\title{
Laboratory examination for Cryptosporidium spp in Scotland
}

\author{
D M Campbell, P A Flanagan
}

\begin{abstract}
Aims: To ascertain the present practices of Scottish microbiological laboratories for examining faecal samples for Cryptosporidium spp and to compare these with practices elsewhere.

Method: A questionnaire was circulated to all 35 Scottish NHS bacteriological laboratories, where human stool samples are subjected to routine microbiological examination, to determine current laboratory testing methods and selection criteria used to examine faecal specimens for Cryptosporidium spp.
\end{abstract}

Results: All laboratories responded, of which the first began testing in 1984 and the last in 1990. At 15 sites all diagnostic stool samples submitted are routinely examined for Cryptosporidium spp. Elsewhere, selection criteria, principally based on age, specific request, and foreign travel, are used. The age distribution of Scottish cases differed significantly from that of the PHLS study of 16 laboratories $(p<0.001)$, probably as a result of the selection criteria used. The phenol-auramine staining procedure is used by 19 laboratories, with modified Ziehl-Neelsen staining used by 14 laboratories; eight used a second, confirmatory method.

Conclusions: Currently applied selection criteria for examination of stools for Cryptosporidium in Scotland do not accord with published epidemiological data. Specimen selection based on age of patient and stool consistency may lead to cases of public health importance being missed. Nationally agreed criteria for testing of human samples based on epidemiological evidence are required.

(f Clin Pathol 1992;45:914-916)

Environmental Health (Scotland) Unit, Glasgow

D M Campbell

Department of Public Health Sciences, University of Edinburgh P A Flanagan

Correspondence to: Dr D M Campbell, Environmental Health (Scotland) Unit, Ruchil Hospital, Glasgow G20 9NB

Accepted for publication 6 April 1992

Cryptosporidium was first identified as a human pathogen in $1976 .^{1}$ Of all the members of this genus of coccidian protozoa, $C$ parvum is believed to be responsible for most cases of clinical disease in both animals and humans. Its importance to public health has been illustrated by several recorded outbreaks of diarrhoeal illness in the United States and the United Kingdom and reinforced by the conclusions of the Group of Experts' report on Cryptosporidium in Water Supplies. ${ }^{23}$

In Scotland NHS microbiology laboratories voluntarily report individual isolations or detections of a range of micro-organisms, on a weekly basis, to the Communicable Diseases (Scotland) Unit (CD[S]U). Cryptosporidium was first routinely reported by Scottish laboratories in 1984 and on 1 January 1989 it became a reportable infection (as distinct from a notifiable disease) in Scotland. ${ }^{4}$ From 1 January 1991 a nationwide active surveillance programme of all reported human cases of cryptosporidiosis has been in operation with the cooperation of microbiologists, consultants in public health medicine, environmental health officers and drinking water quality control staff. As part of this work a survey of laboratory testing methods and selection criteria used to examine human faecal samples for Cryptosporidium was performed.

\section{Methods}

A postal questionnaire was sent to a named medical microbiologist or head of laboratory at each of the 35 NHS general microbiology laboratories in Scotland. A single reminder was issued. Information was sought on when individual laboratories began testing stool samples for Cryptosporidium, current selection criteria, laboratory methods presently used, and any changes in criteria and methods since testing began. The records of $C D[S] U$ were examined to determine the numbers of cases reported each month by individual laboratories between 1984 and 1990. Using Registrar-General (Scotland) population estimates, calculations were made of the populations served by individual or groupings of laboratories. ${ }^{5}$

\section{Results}

Responses were received from 35 (100\%) laboratories; all had been providing a routine microbiological service throughout the period 1984-90. The first laboratories began testing in 1984 with the entirety examining clinical stool samples for Cryptosporidium by 1990 (table 1).

The crude numbers of cases of cryptosporidiosis and rates per 100000 total Scottish population show an apparent large increase in laboratory diagnosis. When the national denominator is corrected for the first month and year that each individual laboratory began testing, the trend in rates is less noticeable (table 2).

All human stool samples submitted are currently tested for Cryptosporidium at 15 sites. The remainder apply a variety of selection criteria, some using more than one; 18 routinely examining stools in response to a specific 
Table 1 Laboratories testing for Cryptosporidium according to year started

\begin{tabular}{llllllll}
\hline & \multicolumn{1}{l}{ Year } & & & & & \\
\cline { 2 - 7 } & '84 & '85 & '86 & '87 & '88 & '89 & '90 \\
\hline $\begin{array}{l}\text { Numbers (\%) of } \\
\text { laboratories }\end{array}$ & $5(14)$ & $11(31)$ & $16(46)$ & $20(57)$ & $27(77)$ & $34(97)$ & $35(100)$ \\
\hline
\end{tabular}

Table 2 Reported cases of cryptosporidiosis by year

\begin{tabular}{|c|c|c|c|c|c|c|c|}
\hline & \multicolumn{7}{|c|}{ Year } \\
\hline & '84 & '85 & '86 & '87 & '88 & '89 & '90 \\
\hline $\begin{array}{l}\text { Cases (Nos) } \\
\text { Rate/100 } 000 \text { (Scotland) } \\
\text { Rate/100 } 000 \text { (UK) }\end{array}$ & $\begin{array}{r}50 \\
1 \\
11\end{array}$ & $\begin{array}{r}265 \\
5 \\
14\end{array}$ & $\begin{array}{r}416 \\
8 \\
15\end{array}$ & $\begin{array}{r}479 \\
9 \\
13\end{array}$ & $\begin{array}{r}546 \\
11 \\
14\end{array}$ & $\begin{array}{r}1240 \\
24 \\
28\end{array}$ & $\begin{array}{r}791 \\
15 \\
17\end{array}$ \\
\hline
\end{tabular}

(Sources: Registrar General for Scotland mid-year population estimates, ${ }^{5} \mathrm{CD}[\mathrm{S}] \mathrm{U}$ laboratory reporting). request, nine where foreign travel is mentioned, 12 only certain age groups, and 16 applying other means of selection-most commonly, stool consistency.

Where a specific age policy exists the commonest (four of 12) is less than 5 years, eight using aged less than 16 . The age related testing policy applied by certain Scottish laboratories produced, for the period 1986-87, a case age distribution significantly different from that reported by the Public Health Laboratory Service Study Group $\left(\chi^{2} 42 \cdot 49 ;\right.$ df8; $\left.p<0.001\right)$ (assuming the underlying population distributions from which the samples were drawn to be similar) which examined all stool samples submitted to 16 laboratories for a range of enteric micro-organisms over the broadly comparable period (table 3$)^{6} .^{6}$

The most frequent "other" criterion applied is based on the consistency of the stool sample submitted-either non-solid, or watery, or liquid samples being examined.

Twelve laboratories had recently modified their testing regimens with five now examining all stools, three widening their inclusion criteria, two altering their testing methods and one changing from universal testing to specific age groups. Two laboratories do not routinely report all diagnosed cases of cryptosporidiosis to $\mathrm{CD}[\mathrm{S}] \mathrm{U}$.

The phenol-auramine staining procedure is used by $19(54 \%)$ laboratories, six of which then proceed to modified Ziehl-Neelsen ( $M$ $\mathrm{Z}-\mathrm{N}$ ) staining. Fourteen (40\%) use the $\mathrm{M} \mathrm{Z-N}$ method, one then using confirmatory monoclonal immunofluorescence and another phenol-auramine staining. Two sites did not supply sufficient details to categorise their techniques.

Table 3 Laboratory reports of cryptosporidiosis by age group (1986-87)

\begin{tabular}{lcc}
\hline Age group & Scotland & PHLS study \\
\hline$<1$ & 81 & 79 \\
$1-4$ & 378 & 480 \\
$5-14$ & 169 & 210 \\
$15-24$ & 63 & 160 \\
$25-34$ & 76 & 155 \\
$35-44$ & 37 & 83 \\
$45-54$ & 11 & 32 \\
$55-64$ & 11 & 12 \\
$>65$ & 14 & 21 \\
\hline
\end{tabular}

\section{Discussion}

This study illustrates the caution that must be exercised when interpreting laboratory reporting data. The total numbers of reports of Cryptosporidium reported from Scottish laboratories increased each year (during 1989 there was one possibly water related outbreak plus a large peak of cases related in time but not in geography), but this can be partially explained by expansion in the number of laboratories examining for, and hence the population at risk of being tested for, the parasite. Interestingly, England and Wales also noted an increase in 1989. ${ }^{3}$ Unfortunately not enough of the laboratories were able to supply records of total numbers of faecal samples examined for Cryptosporidium to allow further inquiry to be made to elucidate whether these increasing rates demonstrate a true trend in incidence of human infection with this parasite.

Current selection criteria applied in Scotland for the examination of diagnostic stool specimens for Cryptosporidium spp do not seem to agree with epidemiological evidence, as demonstrated by the PHLS Study Group. ${ }^{\circ}$ Under policies which applied in 1986-87, 55\% of cases were identified in children aged less than 5 years, with a further $20 \%$ in those between 5 and 14 while the Study Group found $59 \%$ of cases in under 14 year olds. The Scottish age distribution is skewed by a third of laboratories applying an age based criterion. The PHLS survey was a prospective investigation looking at all ages with a standardised methodology; this present study was retrospective and unstandardised, of laboratories using variable selection criteria and methodology. Evidence from outbreaks of cryptosporidiosis suggest that waterborne incidents tend to be associated with a higher number of adult cases than occur in the typical widespread gastroenteritis affecting persons of all ages. ${ }^{3}$ The current Scottish laboratory practice, where $34 \%$ of all laboratories base their specimen selection for routine examination of stools for Cryptosporidium on an age cutoff which excludes adults, may mean that adult initial primary cases in a waterborne outbreak would be missed.

There is no published evidence to support basing selection of specimens for testing on stool consistency. ${ }^{7}$ In the initial clinical stages there may be watery, green or clear, profuse and offensive diarrhoea, with frequency varying from two to 20 motions per day. Subsequently frequent and intermittent looseness may persist for up to a further four weeks. During the infection the number of oocysts excreted may fluctuate with some formed and semi-formed stools containing many oocysts while fluid stools may only have a few. Excretion may continue for prolonged periods in some cases. Determination of risk factors is also difficult, even assuming sufficient information is entered on laboratory request forms. The PHLS Group found that 23\% of their cases reported farm animal contact and a further $26 \%$ indicated the possibility of person to person spread; the remainder were of unknown source. ${ }^{6}$ There was an association 
with foreign travel in $12 \%$ of infections.

Various methods have been described for the detection and identification of oocysts in stool samples. In the mid-1980s studies showed that modified acid-fast staining was the most useful method available. ${ }^{8}$ Since then the use of immunological methods has developed and more recent studies suggest that indirect monoclonal antibody techniques have a $100 \%$ sensitivity and specificity, followed closely by the auramine fluorescence technique. ${ }^{9-1}$ Compared with these, the modified ZiehlNeelsen stains are less satisfactory with, in one study, sensitivity and specificity of $41 \%$ and $52 \%$, respectively. ${ }^{12}$ Those techniques in use in Scottish laboratories are generally similar to those used elsewhere.

It is important that anyone performing surveillance of infection based on routine laboratory reporting data is aware of the following influences on the information: (1) laboratory techniques used and their validity; (2) specimen selection criteria used by individual laboratories; (3) degree of completeness of reporting by laboratories to surveillance centre; (4) any changes to the above during the period under surveillance.

In the case of Cryptosporidium further studies are needed to ascertain the comparative sensitivity and specificity of the different diagnostic techniques. This study supports the findings of the Badenoch Group, that nationally agreed criteria for testing of human stool samples based on epidemiological data are required and that the reporting of all cases by labo- ratories should be encouraged. ${ }^{3}$ The former is being examined by a Cryptosporidium Working Group under the chairmanship of Dr C Roberts, while the latter has been addressed within Scotland by the introduction of the system of reportable infections. ${ }^{4}$

This study would not have been possible without the long established cooperation between $C D[S] U$ and the microbiolog laboratories throughout Scotland. The assistance of all colleagues in completing yet another questionnaire is gratefully acknowledged. The instrument used was piloted initially by $D$ C N Ramsay.

1 Nime FA, Burek JD, Page DL, et al. Acute enterocolitis in a human being infected with the protozoan Cryptosporidium. Gastroenterology 1976;70:592-8.

2 Casemore DP. Epidemiological aspects of human cryptosporidiosis. Epidemiol Infect 1990;104:1-28.

3 Report of the Group of Experts, Chairman-Sir John Badenoch. Cryptosporidium in Water Supplies. London: Badenoch. Crypt
HMSO. 1990.

4 Anon. Scottish surveillance for reportable infections [Editorial]. Lancet 1989;i:397.

5 Registrar-General (Scotland). Annual Reports 1984-1990. Edinburgh: HMSO.

6 Palmer SR, Biffin AH. Cryptosporidiosis in England and Wales: prevalence and epidemiological features. $\mathrm{Br} \mathrm{Med} f$ 1990;300:774-7.

7 Casemore DP. Laboratory methods for diagnosing cryptosporidiosis. f Clin Pathol 1991;44:445-51.

8 Fayer R, Ungar LP. Cryptosporidium spp. and cryptosporidiosis. Microbiol Rev 1986;50:458-83.

9 McLaughin J, Casemore DP, Harrison TB, al Identification of Cryptosporidium spp oocysts by monoclonal tion of Cryptosporidium $\mathrm{spp}$

10 Garcia LS, Brewer TC, Bruckner DA. Fluorescence detection of Cryptosporidium oocysts in human fecal specition of Cryptosporidium oocysts in human fecal specimens by using mor

11 Rusnal J, Hadfield KL, Rhodes MM, et al. Detection of Cryptosporidium oocysts in human fecal specimens by an individual immunofluorescence assay with monoclonal antibodies. $\mathcal{F}$ Clin Microbiol 1989;27:1135-6.

12 Arrowood MJ, Sterling CR. Comparison of conventional staining methods and monoclonal antibody-based methods for Cryptosporidium oocyst detection. $f$ Clin Microbiol 1989;27:1490-5. 